\title{
Simultaneous optimization of replica in multi-cloud simulation
}

\author{
Sunny Bhadlawala*, S.S. Srivastava ${ }^{2}$ \\ ${ }^{1,2}$ Dept. of Computer Science and Engineering AISECT University, Bhopal, India
}

Available online at: www.isroset.org

Received: 15/Mar/2018, Revised: 23/Mar/2018, Accepted: 08/Apr/2018, Online: 30/Apr/ 2018

\begin{abstract}
- in this research paper, we analyze the replication and data partitions, if at any time a master replica has been set to handle all requests, we've talked about servers with high availability and primarily backup plans in their database. On the other hand, if a replication process a request and then redistributes it, it is a multi-primary plan. Transactional replication typically integrates server-to-server scenarios used in the report data from multiple sites would need to integrate high throughput, including improved scalability and availability, Data warehousing and heterogeneous data. Cloud computing is a set of security-control-based technologies and policies that comply with regulatory compliance rules and protect information related to cloud computing, data applications, and infrastructure. To transfer the current server cloud provider to another provider, the security should work according to the following numbers.
\end{abstract}

Keywords-Cloud computing Replica, Data Segmentation, unicast, any cast, multicast, and cloudsim.

\section{INTRODUCTION}

Cloud computing is about distributing computers from remote locations to users.

With Cloud Computing, the user can store their data in the cloud, which is stored in the cloud in the center and can access it from anywhere via the Internet.

The National Institute of Standards and Technology (NIST) defines cloud computing as a model for accessing a common pool of resources (such as networks, servers, storage, applications, and services) of ubiquitous, ondemand, practical network resources.

This can be done quickly and is spent with minimal management effort or interaction with the service provider.

New developments in the field of information technology offer people joy, comfort and convenience.

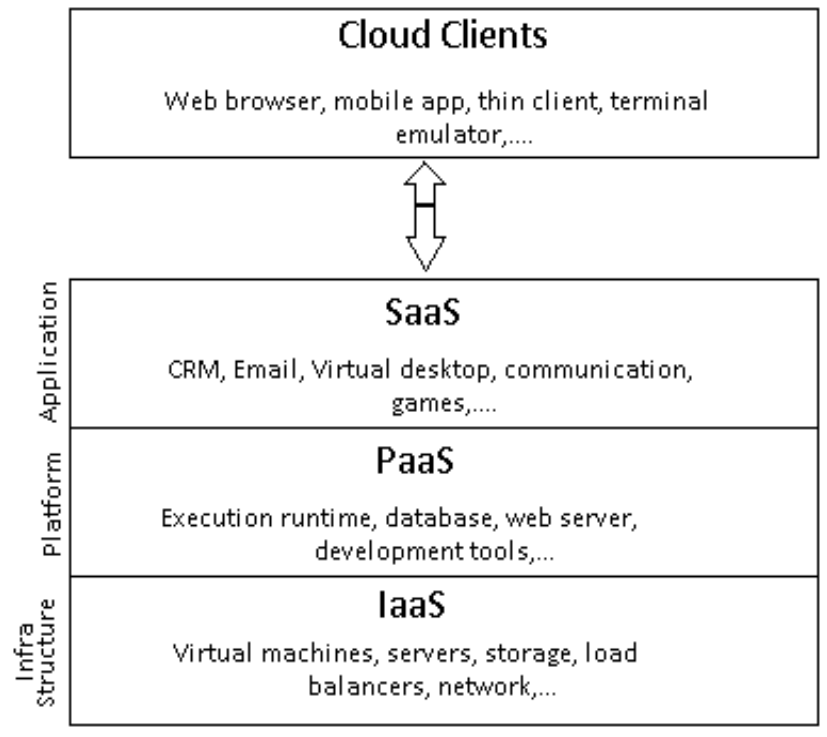

Fig - 1: Cloud general architecture

Cloud computing is one of the latest developments in the IT industry, also known as on-demand computing.

It offers full scalability, reliability, high performance, and relatively low cost, which is a viable solution compared to the dedicated framework. 
This is the application and data centers provided in the form of Internet services in the system hardware that provides these services.

This technique provides the ability to enter a general collection of resources upon request. This money is proving to be very tight for IT departments that want to offer better services under pressure.

Cloud Computing Deployment and Service Models Description Speed Model: Private Cloud, Public Cloud, Community Cloud, And Hybrid Cloud Service Model: Software as a Service (SaaS), Platform as a Service (Pass), And Infrastructure as a Service (IAAS).

When this cloud is made available to the general customer at the user level, it is called a public cloud.

When the client develops its own applications and operates its own internal infrastructure, the private cloud is called.

The integration and consolidation of a public and a private cloud is called Hybrid Cloud.

However, with the many benefits of IT organizations, there are a few cloud issues to consider when deploying.

The key issue is the confidentiality of the security and the confidence that these problems will occur when deploying most public clouds, as the public cloud infrastructure customers do not know how to grow in data storage and the Internet.

In recent years, the advent of cloud computing has shown an interest in various organizations, institutions and users to take advantage of web applications.

The new economic model for the Information Technology (IT) department has revealed that cloud computing has made promises

The model results in a change from an organization that needs to invest substantially in internal IT resources that are managed internally, for a model in which the organization can buy or rent the resources available in the cloud. Payment is in accordance with the use per use in multi-cloud computing by the provider.
Based on the availability of demand for cloud computing resources, it promotes scalability of resources and replication of the same server.

\section{II.PREVIOUSPAPERS STUDY}

The Base Paper ${ }^{[9]}$, Cloud Computing Identity-based Data Storage: Identity-based proxy-re-encryption methods have been proposed to the owner to change the load of managing multiple files on a proxy server.

The existing solutions, however, have many shortcomings. First immigration is determined by the central authority, which makes it impractical to plan.

Second, they are vulnerable to militant attacks. In the end, only these domains (intra-domains) are asked questions.

We note that the identity-based proxy is the primary cloud of the encryption scheme in the cloud computing landscape

However, in this scenario, users can share files in different domains

Therefore, the current solution actually implements this plan does not solve the inspiring landscape while cloud computing.

Therefore, it remains an interesting and challenging research issue protected by the association of attacks and inter-domain domain, inter-domain queries to design identity-based data acquisition system.

In this paper, we propose an identity-based data storage scheme in which intra-domain and inter-domain can both be a question and resist marble attacks.

In addition, access permissions can be set independently of the owner @ 2012 Elsewhere BV.

\section{PROPOSED APPROACH}

This research focuses on a series of strategic issues with multi-cloud, data splitting, security and protocols associated with a successful revolution necessary to ensure a successful process of adoption.

It expands the key to technical factors for adoption, introducing the underlying technology cloud computing, 
and describing various deployment and deployment models for cloud computing.

There are many challenges for the introduction of cloud computing, these are the challenges: security challenges,

When we talk about a copy of the cloud-based server, it's always tied to legal issues and compliance challenges and organizational challenges.

All this is related to the challenges that are the trust between customers and suppliers.

With cloud computing, businesses need to rely on vendors to manage their IT resources and data

As the Trust will be an important driver for the adoption of cloud computing, it will focus specifically on this research in order to meet the challenges of organizations while encouraging the adoption of various pointers.

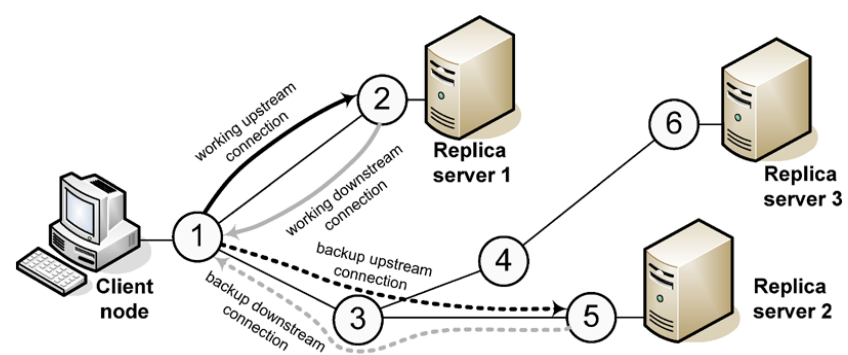

Fig - 2 cloud replica servers.

Server replication for a specific virtual machine on the primary host server begins by creating a similar virtual machine in the secondary replication start point.

After the initial replication, the server replicates a log file for the virtual machine.

Depending on the frequency of replication, the log file will be played in reverse order in the copy. This protocol and the reverse order mean that the most recent changes are replicated and replicated asynchronously.

If the replication does not occur in accordance with the expected frequency, a warning is issued.

Here you set up the renationalization settings for a virtual machine. It can be done manually, automatically or automatically within a specific program. Since our research is based on the protocol, in order to move the main replication to another, we must follow the constraints described in the equations.

The transmission is based on unicast and anycast with nonexistent networks.

The UAFA also knows the unicast and anycast flow which defined in the networks.

$\min \varphi=\sum_{r \in D} \quad \sum_{h \in A} \varepsilon_{\mathrm{h}}\left(x_{r . h}+y_{r . h}\right) \ldots(1)$

Based on the subject to

$\sum_{h \in\left\{h: a_{h}=(n, j) \in A, j \in N, j \neq n\right\}} x_{r . h}-\sum_{h \in\left\{h: a_{h}=(i, n) \in A, i \in N, i \neq n\right\}} x_{r . h}$

$=\left\{\begin{array}{l}1, \text { if } n=s_{r} \\ -1, \text { if } n=t_{r} \\ 0 \text { otherwise }\end{array}, \forall n \in D^{U N},\right.$.

$\begin{aligned} & \sum_{h \in\left\{h: a_{h}=(n, j) \in A, j \in N, j \neq n\right\}} y_{r . h}-\sum_{h \in\left\{h: a_{h}=(i, n) \in A, i \in N, i \neq n\right\}} y_{r . h} \\ = & \left\{\begin{array}{c}1, \text { if } n=s_{r} \\ -1, \text { if } n=t_{r} \\ 0 \quad \text { otherwise }\end{array}\right.\end{aligned}$

$\sum_{h \in\left\{h: a_{h}=(n, j) \in A, j \in N, j \neq n\right\}} x_{r . h}-\sum_{h \in\left\{h: a_{h}=(i, n) \in A, i \in N, i \neq n\right\}} x_{r . h}$

$=\left\{\begin{array}{ll}-1, & \text { if } n=t_{r} \\ Z_{r, n} & \text { if } n \neq t_{r}\end{array} \forall r \in D^{D S}, \forall n \in N \ldots .\right.$.

$\sum_{h \in\left\{h: a_{h}=(n, j) \in A, j \in N, j \neq n\right\}} y_{r . h}-\sum_{h \in\left\{h: a_{h}=(i, n) \in A, i \in N, i \neq n\right\}} y_{r . h}$

$= \begin{cases}-1, & \text { if } n=t_{r} \\ \vartheta_{r, n}, & \text { if } n \neq t_{r} \forall r \in D^{D S}, \forall n \in N \ldots\end{cases}$

$\sum_{h \in\left\{h: a_{h}=(n, j) \in A, j \in N, j \neq n\right\}} x_{r . h}-\sum_{h \in\left\{h: a_{h}=(i, n) \in A, i \in N, i \neq n\right\}} x_{r . h}$ 
$=\left\{\begin{array}{l}-1, \text { if } n=s_{r} \\ -Z_{r, n} \text { if } n \neq s_{r} \forall r \in D^{U S}, \forall n \in N\end{array}\right.$

$\sum_{h \in\left\{h: a_{h}=(n, j) \in A, j \in N, j \neq n\right\}} y_{r . h}-\sum_{h \in\left\{h: a_{h}=(i, n) \in A, i \in N, i \neq n\right\}} y_{r . h}$

$=\left\{\begin{array}{l}1, \text { if } n=s_{r} \\ -\vartheta_{r, n} \text { if } n \neq s_{r} \forall r \in D^{U S}, \forall n \in N\end{array}\right.$

$z_{r, n} \leq u_{n} \forall r \in D^{A N}, \forall n \in N$

$\vartheta_{r, n} \leq u_{n} \forall r \in D^{A N}, \forall n \in N$

$z_{r, n} \leq z_{\tau(r), n} \forall r \in D^{D S}, \forall n \in N \ldots$

$\vartheta_{r, n} \leq \vartheta_{\tau(r), n} \forall r \in D^{D S}, \forall n \in N$

$\sum_{n \in N} \quad z_{r, n}=1 \forall r \in D^{A N} \ldots$

$\sum_{n \in N} \quad \vartheta_{r, n}=1 \forall r \in D^{A N}$

$\sum_{r \in D} \quad\left(x_{r, h}+y_{r, h}\right) \leq 1 \forall h \in \mid A$. $\left(x_{r, h}+y_{r, h}\right) \leq 1 \forall r \in D, \forall h \in A \ldots$.

$\left(x_{r, h}+y_{\tau(r), h}\right) \leq 1 \forall r \in D^{A N}, \forall h \in A \ldots$

The linear cost of the overall flow which allocated in the networks based on the constraints and the stream demands (d) if current node ' $n$ ' is selected as a working replica of demand ' $\mathrm{d}$ '. The replica node can be selected as anodes $n \in N$ host replica $\left(u_{n}=1\right)$.

In the equation 1 to 16 defined there is no coupling between the working replica servers.

Two cases are possible for each anycast demand:

Working replica servers are located at different nodes.

Working replica servers are located at the same node. For ease of reference defined 1 to 16 as Any Type Replica (ATR) model.

IV. SIMULATION

\begin{tabular}{|c|c|c|c|c|c|c|c|}
\hline User & $\begin{array}{c}\text { Cloudlet } \\
\text { ID }\end{array}$ & Status & $\begin{array}{c}\text { Data } \\
\text { Centre ID }\end{array}$ & VM & Time & Start Time & $\begin{array}{l}\text { Finish } \\
\text { Time }\end{array}$ \\
\hline 5 & 0 & SUCCESS & 2 & 1 & 160 & 0 & 160 \\
\hline 5 & 0 & SUCCESS & 3 & 1 & 80 & 160 & 240 \\
\hline 5 & 0 & SUCCESS & 4 & 1 & 240 & 240 & 480 \\
\hline 5 & 0 & SUCCESS & 5 & 1 & 60 & 480 & 540 \\
\hline 6 & 1 & SUCCESS & 2 & 1 & 160 & 1 & 161 \\
\hline 6 & 1 & SUCCESS & 3 & 1 & 80 & 161 & 241 \\
\hline 6 & 1 & SUCCESS & 4 & 1 & 240 & 241 & 481 \\
\hline 6 & 1 & SUCCESS & 5 & 1 & 160 & 481 & 641 \\
\hline - & - & - & - & - & - & - & - \\
\hline - & - & - & - & - & . & - & - \\
\hline - & - & $\cdot$ & - & - & $\cdot$ & - & . \\
\hline $\mathrm{n}$ & $\mathrm{n}^{\text {th }}$ & SUCCESS & $n+1$ & 1 & $\mathrm{t}+\mathrm{t}^{\mathrm{n}}$ & $\mathrm{t}$ & $t^{n}$ \\
\hline
\end{tabular}

Table - I: Cloud simulation

\section{CONCLUSION}

In this research paper, we have implemented a proposed structure for the replica of the servers and its data for or when required.
Now a day's it's a very important when a huge number of server required and should require access number of resources which cannot available on the same site or server. 
And as based on the cloud computing structure it also available on demand without consuming much time and at a time it can shift to the different location or site so based on this research we concluded that we are extended cloud elasticity and reliability for availability as and when required.

\section{REFERENCES}

[1]. Cong Wang, Sherman S.M. Chow, Qian Wang, Kui Ren, Wenjing Lou, "Privacy-Preserving Public Auditing for Secure Cloud Storage", IEEE TRANSACTIONS ON COMPUTERS, VOL. 62, NO. 2, FEBRUARY 2013

[2]. Nancy J. King, V.T. Raja, "Protecting the privacy and security of sensitive customer data in the cloud", computer law \& security review28(2012)308e319

[3]. Abhishek Mohta ,Ravi Kant Sahu,Lalit Kumar Awasthi, "Robust Data Security for Cloud while using Third Party Auditor",International Journal of Advanced Research in Computer Science and Software Engineering, Volume 2, Issue 2, February 2012

[4]. Ronnie D. Caytiles and Sunguk Lee, "Security Considerations for Public Mobile Cloud Computing ", international Journal of Advanced Science and Technology Vol. 44, July, 2012

[5]. Kangchan Lee, "Security Threats in Cloud Computing Environments", International Journal of Security and Its Applications Vol. 6, No. 4, October, 2012

[6]. Abdul Nasir Khan, M.L. Mat Kiah, Samee U. Khan, Sajjad A. Madani, "Towards secure mobile cloud computing: A survey", Contents lists available at SciVerseScienceDirect Future Generation Computer Systems

[7]. Cong Wang, Qian Wang, Kui Ren, Ning Cao, Wenjing Lou, "Towards Secure and Dependable Storage Services in Cloud Computing", IEEE Transactions on Cloud Computing Date of Publication: April-June 2012 Volume: 5 , Issue: 2

[8]. M. K. F. H. Judith Hurwitz, Robin Bloor, "Cloud computing for dummies," http://www.dummies.com/how-to/content/ cloud-computing-standards-organizations.html.

[9]. J. Han, W. Susilo, and Y. Mu, "Identity-based data storage in cloud computing," Future Generation Computer Systems, vol. 29, no. 3, pp. 673-681, 2013.

[10]. L. M. Kaufman, "Data security in the world of cloud computing," Security \& Privacy, IEEE, vol. 7, no. 4, pp. 6164, 2009.

[11]. C. Cachin, I. Keidar, and A. Shraer, "Trusting the cloud," AcmSigact News, vol. 40, no. 2, pp. 81-86, 2009.

[12]. P. Kumar and H. S. Arri, "Data location in cloud computing," International Journal for Science and Emerging Technologies with Latest Trends, vol. 5, no. 1, pp. 24-27, 2013.

[13]. "IBM-Uncovers-Encryption-Scheme-That-Could-ImproveCloud-Security," http://www.eweek.com/c/a/Security/IBMUncovers-Encryption-Scheme-/ That-Could-Improve-CloudSecurity-Spam-Filtering-135413.

[14]. K. P. Puttaswamy, C. Kruegel, and B. Y. Zhao, "Silverline: toward data confidentiality in storageintensive cloud applications," in Proceedings of the 2nd ACM Symposium on Cloud Computing, p. 10, 2011.

[15]. A. S. Team et al., "Amazon s3 availability event: July 20, 2008," See: http://status. aws. amazon. com/s3-20080720. html, 2008.

[16]. K. Sharma and K. R. Singh, "Online data back-up and disaster recovery techniques in cloud computing: A review,"
International Journal of Engineering and Innovative Technology (IJEIT), vol. 2, no. 5, pp. 249-254, 2012.

[17]. EmanM.Mohamed, Hatem S. Abdelkader, "Enhanced Data Security Model for Cloud Computing", The 8th International Conference on INFOrmatics and Systems (INFOS2012) - 1416 May

[18]. K. R. C. Wang, Q. Wang and W. Lou, "Ensuring data storage security in cloud computing," in Proc. 17th International Workshop on Quality of Service (IWQoS '09), pp. 1-9, 2009. N. I. of Standards and Technology (NIST). http://www.nist.gov/itl/cloud/. 